\title{
EIGENVALUE DECAY OF OPERATORS ON HARMONIC FUNCTION SPACES
}

\author{
OSCAR F. BANDTLOW AND CHO-HO CHU
}

\begin{abstract}
Let $\Omega$ be an open set in $\mathbb{R}^{d}(d>1)$ and $h(\Omega)$ the Fréchet space of harmonic functions on $\Omega$. Given a bounded linear operator $L: h(\Omega) \rightarrow h(\Omega)$, we show that its eigenvalues $\lambda_{n}$, arranged in decreasing order and counting multiplicities, satisfy $\left|\lambda_{n}\right| \leq K \exp \left(-c n^{1 /(d-1)}\right)$, where $K$ and $c$ are two explicitly computable positive constants.
\end{abstract}

\section{INTRODUCTION}

In his celebrated memoirs [7], Grothendieck showed that the eigenvalues, ordered by magnitude and counting algebraic multiplicities, of every bounded operator on a quasi-complete nuclear space decrease rapidly [7, Chap II, $§ 2$, No. 4, Corollaire 3]. He also remarked in [7, Chap II, §2, No. 4, Remarque 9] that this result could be improved for certain spaces including the space $\mathcal{H}(\Omega)$ of holomorphic functions on a domain $\Omega$ in $\mathbb{C}^{d}$. Indeed, he showed that the eigenvalues

$$
\left|\lambda_{1}\right| \geq\left|\lambda_{2}\right| \geq \cdots \geq\left|\lambda_{n}\right| \geq \cdots
$$

of a bounded operator on $\mathcal{H}(\Omega)$ satisfy

$$
\lambda_{n}=O\left(\exp \left(-c n^{1 / d}\right)\right)
$$

for some positive constant $c$. We note that Grothendieck originally asserted that $\lambda_{n}=O(\exp (-c n))$ although his arguments actually yield the above dimensiondependent decay (see [5, Appendix A] for a short alternative proof of (10)).

In this paper, we show that a decay of this type also occurs for the eigenvalues of bounded operators on the space $h(\Omega)$ of harmonic functions on a non-empty open set $\Omega$ in $\mathbb{R}^{d}(d>1)$. More precisely, we shall show that there are positive constants $K$ and $c$, such that

$$
\left|\lambda_{n}\right| \leq K \exp \left(-c n^{1 /(d-1)}\right) .
$$

The main ingredient in our proof is to show, by using properties of spherical harmonics, that the singular values of certain natural embeddings of harmonic Bergman spaces on balls decay at a stretched exponential rate (see Proposition 3.3. which, in fact, yields the precise asymptotics of the singular value decay of these embeddings). After extending this result to embeddings associated with more general open sets (see Theorem 4.5) some abstract operator theoretic techniques discussed in Section 2 then yield the main result in Theorem 5.3. This method

Date: February 26, 2009.

1991 Mathematics Subject Classification. 47B38, 47B07, 47B06, 46E10, 31B05.

Key words and phrases. Bounded operator, harmonic function space, eigenvalue asymptotic. 
also yields explicitly computable estimates for the constants $K$ and $c$ occurring in (2), which will be illustrated by a concrete example at the end of this article.

Notation 1.1. Let $H_{1}$ and $H_{2}$ be Hilbert spaces. Throughout, we use $\mathcal{L}\left(H_{1}, H_{2}\right)$ to denote the Banach space of bounded linear operators from $H_{1}$ to $H_{2}$, equipped with the usual norm, and $\mathcal{S}_{\infty}\left(H_{1}, H_{2}\right) \subset \mathcal{L}\left(H_{1}, H_{2}\right)$ to denote the closed subspace of compact operators from $H_{1}$ to $H_{2}$. We shall often write $\mathcal{L}$ or $\mathcal{S}_{\infty}$ if the Hilbert spaces $H_{1}$ and $H_{2}$ are understood.

For $A \in \mathcal{S}_{\infty}(H, H)$ we let $\lambda(A)=\left\{\lambda_{n}(A)\right\}_{n=1}^{\infty}$ denote the sequence of eigenvalues of $A$, each eigenvalue repeated according to its algebraic multiplicity, and ordered by magnitude, so that $\left|\lambda_{1}(A)\right| \geq\left|\lambda_{2}(A)\right| \geq \ldots$. We also write $|\lambda(A)|$ for the sequence $\left\{\left|\lambda_{n}(A)\right|\right\}_{n=1}^{\infty}$.

Similarly, for $A \in \mathcal{S}_{\infty}\left(H_{1}, H_{2}\right)$, we use $s(A)=\left\{s_{n}(A)\right\}_{n=1}^{\infty}$, where

$$
s_{n}(A)=\sqrt{\lambda_{n}\left(A^{*} A\right)} \quad(n \in \mathbb{N}),
$$

to denote the sequence of singular values of $A$.

\section{EXPONENTIAL CLASSES}

In this section we consider classes of compact operators whose singular values decay exponentially of a particular order and list some of their properties. We start by introducing exponential classes of complex sequences. Let $a>0$ and $\alpha>0$. We define

$$
\mathcal{E}(a, \alpha):=\left\{x \in \mathbb{C}^{\mathbb{N}}\left|\sup _{n \in \mathbb{N}}\right| x_{n} \mid \exp \left(a n^{\alpha}\right)<\infty\right\} .
$$

Then $\mathcal{E}(a, \alpha)$ is a complex Banach space with norm

$$
|x|_{a, \alpha}:=\sup _{n \in \mathbb{N}}\left|x_{n}\right| \exp \left(a n^{\alpha}\right),
$$

which we call exponential class of sequences of type $(a, \alpha)$. The set

$$
\mathcal{E}(\alpha):=\bigcup_{a>0} \mathcal{E}(a, \alpha)
$$

will be referred to as exponential class of sequences of type $\alpha$.

Definition 2.1. Let $H_{1}$ and $H_{2}$ be Hilbert spaces, which, to avoid trivialities, we assume to be infinite dimensional. For $a, \alpha>0$, we define

$$
E\left(a, \alpha ; H_{1}, H_{2}\right):=\left\{\left.A \in \mathcal{S}_{\infty}\left(H_{1}, H_{2}\right)|| A\right|_{a, \alpha}:=\sup _{n \in \mathbb{N}} s_{n}(A) \exp \left(a n^{\alpha}\right)<\infty\right\},
$$

which is called exponential class of operators of type $(a, \alpha)$. We refer to the number $|A|_{a, \alpha}$ as $(a, \alpha)$-gauge or simply gauge of $A$. The set

$$
E\left(\alpha ; H_{1}, H_{2}\right):=\bigcup_{a>0} E\left(a, \alpha ; H_{1}, H_{2}\right)
$$

is called the exponential class of operators of type $\alpha$. It consists of compact operators between $H_{1}$ and $H_{2}$ whose singular values decay at a stretched exponential rate with stretching exponent $\alpha$. 
Whenever the Hilbert spaces are clear from the context, we suppress reference to them and simply write $E(a, \alpha)$ instead of $E\left(a, \alpha ; H_{1}, H_{2}\right)$ and similarly for $E(\alpha)$.

We now collect a number of properties of the classes $E(a, \alpha)$ which will be used later.

Proposition 2.2. Let $\alpha, a, a_{1}, \ldots, a_{N}>0$.

(i) If $A, C \in \mathcal{L}$ and $B \in E(a, \alpha)$, then

$$
A B C \in E(a, \alpha) \quad \text { and } \quad|A B C|_{a, \alpha} \leq\|A\||B|_{a, \alpha}\|C\| .
$$

(ii) Let $A_{n} \in E\left(a_{n}, \alpha\right)$ for $1 \leq n \leq N$ and let $A=\sum_{n=1}^{N} A_{n}$. Then

$$
A \in E\left(a^{\prime}, \alpha\right) \text { with }|A|_{a^{\prime}, \alpha} \leq N \max _{1 \leq n \leq N}\left|A_{n}\right|_{a_{n}, \alpha}
$$

where $a^{\prime}:=\left(\sum_{n=1}^{K} a_{n}^{-1 / \alpha}\right)^{-\alpha}$. In particular

$$
E\left(a_{1}, \alpha\right)+\cdots+E\left(a_{N}, \alpha\right) \subset E\left(a^{\prime}, \alpha\right)
$$

and the above inclusion is sharp in the sense that

$$
E\left(a_{1}, \alpha\right)+\cdots+E\left(a_{N}, \alpha\right) \not \subset E(b, \alpha)
$$

for $b>a^{\prime}$

(iii) If $A \in E(a, \alpha)$, then

$$
\lambda(A) \in \mathcal{E}(a /(1+\alpha), \alpha) \text { with }|\lambda(A)|_{a /(1+\alpha), \alpha} \leq|A|_{a, \alpha} .
$$

The result is sharp in the sense that there is an operator $A \in E(a, \alpha)$ such that $\lambda(A) \notin \mathcal{E}(b, \alpha)$ whenever $b>a /(1+\alpha)$.

Proof. See [4, Prop. 2.5, 2.8, and 2.10].

Remark 2.3. Note that $E\left(a, \alpha ; H_{1}, H_{2}\right)$ is not a linear space. In order to see this assume for simplicity that $H_{1}=H_{2}=\ell^{2}$. Let $\sigma_{n}=\exp \left(-a n^{\alpha}\right)$ and let $A$ and $B$ be the diagonal operators $A=\operatorname{diag}\left(\sigma_{1}, 0, \sigma_{2}, 0, \ldots\right)$ and $B=\operatorname{diag}\left(0, \sigma_{1}, 0, \sigma_{2}, \ldots\right)$. Then $A, B \in E(a, \alpha)$ with $|A|_{a, \alpha}=|B|_{a, \alpha}=1$ but $A+B=\operatorname{diag}\left(\sigma_{1}, \sigma_{1}, \sigma_{2}, \sigma_{2}, \ldots\right) \notin$ $E(a, \alpha)$. Essentially the same construction can be used to deal with the case of arbitrary (infinite-dimensional) spaces $H_{1}$ and $H_{2}$.

The fact that $E(a, \alpha)$ is not a linear space also follows from assertion (ii) of the previous proposition, which implies that $E(a, \alpha)+E(a, \alpha) \subset E\left(2^{-\alpha} a, \alpha\right)$, but $E(a, \alpha)+E(a, \alpha) \not \subset E(a, \alpha)$, because $2^{-\alpha} a<a$.

\section{HARMONIC BERGMAN SPACES AND CANONICAL IDENTIFICATIONS}

To pave the way for the main result, our objective in this section will be to show that certain natural embeddings of harmonic Bergman spaces have singular values which decay at a stretched exponential rate.

In the sequel, all open sets in $\mathbb{R}^{d}$ are non-empty. Let $\Omega \subset \mathbb{R}^{d}$ be an open set and let $L^{2}(\Omega)$ be the Lebesgue space of complex-valued, square-integrable functions on $\Omega$ with respect to Lebesgue measure $d x$ on $\mathbb{R}^{d}$, equipped with the usual norm. Let $\Delta$ be the Laplace operator and let

$$
h^{2}(\Omega):=\left\{f \in L^{2}(\Omega) \mid \Delta f=0\right\}
$$


be the harmonic Bergman space over $\Omega$, which is a separable Hilbert space with inner product

$$
(f, g)_{h^{2}(\Omega)}=\int_{\Omega} f(x) \overline{g(x)} d x \quad\left(f, g \in h^{2}(\Omega)\right) .
$$

We refer to [2] for more details about $h^{2}(\Omega)$.

Suppose that $\Omega_{1}, \Omega_{2} \subset \mathbb{R}^{d}$ are open and that $\Omega_{2} \subset \Omega_{1}$. By restriction to $\Omega_{2}$ every element in $h^{2}\left(\Omega_{1}\right)$ can also be considered as an element of $h^{2}\left(\Omega_{2}\right)$. This restriction yields a linear transformation $J: h^{2}\left(\Omega_{1}\right) \rightarrow h^{2}\left(\Omega_{2}\right)$, called the canonical identification. If $\Omega_{1}$ is connected, then the canonical identification is injective and hence a proper embedding of $h^{2}\left(\Omega_{1}\right)$ in $h^{2}\left(\Omega_{2}\right)$.

It is obvious that $J$ is continuous. Under stronger assumptions about $\Omega_{1}$ and $\Omega_{2}$, more can be said about $J$. We require the following definition.

Definition 3.1. Let $\Omega_{1}, \Omega_{2}$ be open subsets of $\mathbb{R}^{d}$. If $\bar{\Omega}_{2}$ is a compact subset of $\Omega_{1}$ then we say that $\Omega_{2}$ is compactly contained in $\Omega_{1}$, and write $\Omega_{2} \subset \Omega_{1}$.

It turns out that if $\Omega_{2} \mathbb{C} \Omega_{1}$ then $J: h^{2}\left(\Omega_{1}\right) \hookrightarrow h^{2}\left(\Omega_{2}\right)$ is a compact operator 1 In fact rather more is true: $J \in E(1 /(d-1))$. The proof of this result requires a certain amount of preparation and will be presented in the next section.

In this section we shall be content with proving this result for the case where $\Omega_{1}$ and $\Omega_{2}$ are concentric balls, in which case the rate of decay can be identified precisely.

Notation 3.2. We denote by $B_{r, x}$ the ball with radius $r$ centred at $x$, with respect to the Euclidean metric. We use $B_{r}$ as a short-hand for $B_{r, 0}$. Given a ball $B=B_{r, x}$ and $\gamma>0$, we use the symbol

$$
B(\gamma):=B_{\gamma r, x}
$$

for the $\gamma$-dilation of the ball. define

As usual, given $x=\left(x_{1}, \ldots, x_{d}\right) \in \mathbb{R}^{d}$ and a multi-index $\alpha=\left(\alpha_{1}, \ldots, \alpha_{d}\right)$, we

$$
x^{\alpha}=x_{1}^{\alpha_{1}} \cdots x_{d}^{\alpha_{d}} \quad \text { and } \quad|\alpha|=\alpha_{1}+\cdots+\alpha_{d} .
$$

A homogeneous harmonic polynomial of degree $k$ in $d$ dimensions is a polynomial $p: \mathbb{R}^{d} \rightarrow \mathbb{C}$ of the form

$$
p(x)=\sum_{|\alpha|=k} c_{\alpha} x^{\alpha} \quad\left(x \in \mathbb{R}^{d}\right),
$$

which is also a harmonic function. The restriction of $p$ to the unit sphere $S$ in $\mathbb{R}^{d}$ is called a spherical harmonic of degree $k$. The number $N_{d}(k)$ of linearly independent spherical harmonics of degree $k$ in $d$ dimensions is given by the power series

$$
\frac{1+x}{(1-x)^{d-1}}=\sum_{k=0}^{\infty} N_{d}(k) x^{k} \quad(|x|<1)
$$

\footnotetext{
${ }^{1}$ To see this, note that $J\left(h^{2}\left(\Omega_{1}\right)\right)$ is contained in the Banach space $C^{b}\left(\Omega_{2}\right)$ of bounded continuous functions on $\Omega_{2}$ and $J: h^{2}\left(\Omega_{1}\right) \rightarrow C^{b}\left(\Omega_{2}\right)$ has closed graph. Hence $\left\{J f:\|f\|_{2} \leq 1\right\}$ is uniformly bounded on $\Omega_{2}$ and therefore a normal family in $h^{2}\left(\Omega_{2}\right)$ (cf. [2, Theorem 2.6]).
} 
(see [8, Lemma 3]). Hence

$$
N_{d}(k)=\left(\begin{array}{c}
k+d-1 \\
d-1
\end{array}\right)-\left(\begin{array}{c}
k+d-3 \\
d-1
\end{array}\right)
$$

(see also [2, Proposition 5.17]). We now define $h_{d}(k)=N_{d}(0)+\cdots+N_{d}(k)$, which is the number of linearly independent homogeneous harmonic polynomials of degree at most $k$ in $d$ dimensions. It follows that

$$
h_{d}(k)=\sum_{l=0}^{k}\left(\left(\begin{array}{c}
l+d-1 \\
d-1
\end{array}\right)-\left(\begin{array}{c}
l+d-3 \\
d-1
\end{array}\right)\right)=\left(\begin{array}{c}
k+d \\
d
\end{array}\right)-\left(\begin{array}{c}
k+d-2 \\
d
\end{array}\right) .
$$

We note that $h_{d}(0)=1$ and define $h_{d}(-1)=0$.

Proposition 3.3. Let $B \subset \mathbb{R}^{d}$ be a ball and $\gamma>1$. Then the singular values of the canonical identification

$$
J: h^{2}(B(\gamma)) \hookrightarrow h^{2}(B)
$$

are given by

$$
s_{n}(J)=\gamma^{-\left(k+\frac{d}{2}\right)}
$$

for $h_{d}(k-1)<n \leq h_{d}(k)$ and $k \in \mathbb{N} \cup\{0\}$.

Proof. By translation invariance of the Lebesgue measure, we may assume that $B$ is centred at the origin, say, $B=B_{r}$. The proof relies on the fact that $h^{2}\left(B_{r}\right)$ and $h^{2}\left(B_{\gamma r}\right)$ have a common complete orthogonal system consisting of homogeneous harmonic polynomials. To see this we first note that the linear span of homogeneous harmonic polynomials is dense in $h^{2}\left(B_{s}\right)$ for every $s>0$ [2, Lemma 8.8]. Let now $f$ and $g$ be homogeneous harmonic polynomials of degree $n$ and $m$ respectively. Then by the polar-coordinate formula for integration in $\mathbb{R}^{d}$ (cf. [10, p. 150]), we have

$$
\begin{aligned}
(f, g)_{h^{2}\left(B_{r}\right)} & =d \operatorname{Vol}\left(B_{1}\right) \int_{0}^{r} \rho^{d-1} \int_{S} f(\rho \xi) \overline{g(\rho \xi)} d \sigma(\xi) d \rho \\
& =d \operatorname{Vol}\left(B_{1}\right) \int_{0}^{r} \rho^{d-1+n+m} \int_{S} f(\xi) \overline{g(\xi)} d \sigma(\xi) d \rho
\end{aligned}
$$

where $S$ is the unit sphere in $\mathbb{R}^{d}$ and $\sigma$ the normalised surface measure on $S$. Therefore

$$
(f, g)_{h^{2}\left(B_{r}\right)}=d \operatorname{Vol}\left(B_{1}\right) \frac{r^{d+n+m}}{d+n+m} \int_{S} f(\xi) \overline{g(\xi)} d \sigma(\xi) .
$$

Since $\int_{S} f \bar{g} d \sigma=0$ whenever $n \neq m$ [2, Theorem 5.3], the Gram-Schmidt orthogonalisation process yields an orthonormal basis for $h^{2}\left(B_{r}\right)$, consisting of homogeneous harmonic polynomials. Observe now that by (3) we have

$$
(f, g)_{h^{2}\left(B_{\gamma r}\right)}=\gamma^{d+n+m}(f, g)_{h^{2}\left(B_{r}\right)}
$$

for any two homogeneous harmonic polynomials $f$ and $g$ of degree $n$ an $m$ respectively. In particular $(f, g)_{h^{2}\left(B_{\gamma r}\right)}=0$ whenever $(f, g)_{h^{2}\left(B_{r}\right)}=0$. This shows that $h^{2}\left(B_{r}\right)$ and $h^{2}\left(B_{\gamma r}\right)$ have a common complete orthogonal system consisting of homogeneous harmonic polynomials. 
In order to see that the canonical identification $J: h^{2}\left(B_{\gamma r}\right) \hookrightarrow h^{2}\left(B_{r}\right)$ has the desired properties, note that by (4) we have

$$
\left(J^{*} J f, g\right)_{h^{2}\left(B_{\gamma r}\right)}=(J f, J g)_{h^{2}\left(B_{r}\right)}=\gamma^{-(d+n+m)}(f, g)_{h^{2}\left(B_{\gamma r}\right)} .
$$

This implies that $J^{*} J$ is diagonal with respect to the orthonormal basis of homogeneous harmonic polynomials. Its eigenvalues therefore belong to the set $\left\{\gamma^{-(2 k+d)} \mid k \in \mathbb{N} \cup\{0\}\right\}$. Consequently, the singular values of $J$ belong to the set $\left\{\gamma^{-\left(k+\frac{d}{2}\right)} \mid k \in \mathbb{N} \cup\{0\}\right\}$. As there are $N_{d}(k)$ linearly independent homogeneous harmonic polynomials of degree $k$, the value $\gamma^{-\left(k+\frac{d}{2}\right)}$ occurs with multiplicity $N_{d}(k)$. If we order the orthonormal basis by degrees, then we have

$$
s_{n}(J)=\gamma^{-\left(k+\frac{d}{2}\right)}
$$

for $h_{d}(k-1)<n \leq h_{d}(k)$.

In order to study the singular value asymptotics of the canonical identification $J$ obtained in the previous proposition, we require the following lemma.

Lemma 3.4. Let $d \in \mathbb{N}$ and let $a_{1}, \ldots, a_{d} \geq 0$. Then

$$
\sup _{x \geq 0} \prod_{k=1}^{d}\left(x+a_{k}\right)^{1 / d}-x=\lim _{x \rightarrow \infty} \prod_{k=1}^{d}\left(x+a_{k}\right)^{1 / d}-x=\frac{1}{d} \sum_{k=1}^{d} a_{k} .
$$

Proof. The case $d=1$ of (5) is clearly true, so suppose $d \geq 2$. Define

$$
h(x)=\prod_{k=1}^{d}\left(x+a_{k}\right)^{1 / d}-x \quad(x \geq 0) .
$$

Then $h$ is an increasing function. Indeed, we have

$$
h^{\prime}(x)=\frac{1}{d}\left(\prod_{k=1}^{d}\left(x+a_{k}\right)^{-1+1 / d}\right) \sum_{k=1}^{d} \prod_{\substack{l=1 \\ l \neq k}}^{d}\left(x+a_{l}\right)-1
$$

where

$$
\frac{1}{d} \sum_{k=1}^{d} \prod_{\substack{l=1 \\ l \neq k}}^{d}\left(x+a_{l}\right) \geq \prod_{k=1}^{d}\left(x+a_{k}\right)^{1-1 / d}
$$

since

$$
\frac{1}{d} \sum_{k=1}^{d}\left(x+a_{k}\right)^{-1} \geq \prod_{k=1}^{d}\left(x+a_{k}\right)^{-1 / d}
$$

by the arithmetic-geometric mean inequality. Thus $h^{\prime}(x) \geq 0$ for $x \geq 0$.

To complete the proof of (5), observe that

$$
\lim _{x \rightarrow \infty} h(x)=\lim _{t \downarrow 0} t^{-1}\left(\prod_{k=1}^{d}\left(1+a_{k} t\right)^{1 / d}-1\right)=\frac{1}{d} \sum_{k=1}^{d} a_{k}
$$

by l'Hôpital's rule. 
Proposition 3.5. Let $d \geq 2$ and $B \subset \mathbb{R}^{d}$ be a ball. Given $\gamma>1$, the canonical embedding

$$
J: h^{2}(B(\gamma)) \hookrightarrow h^{2}(B)
$$

satisfies

$$
J \in E(c, 1 /(d-1)), \quad \text { where } c=\left(\frac{(d-1) !}{2}\right)^{1 /(d-1)} \log \gamma
$$

and

$$
|J|_{c, 1 /(d-1)}=\gamma^{-1 / 2}
$$

In other words, its singular value sequence $s(J)$ has the following asymptotics:

$$
\lim _{n \rightarrow \infty} \frac{\log \left|\log s_{n}(J)\right|}{\log n}=\frac{1}{d-1}
$$

$$
\begin{gathered}
\lim _{n \rightarrow \infty} \frac{\log s_{n}(J)}{n^{1 /(d-1)}}=-\left(\frac{(d-1) !}{2}\right)^{1 /(d-1)} \log \gamma \\
\sup _{n \in \mathbb{N}}\left(\log s_{n}(J)+\left(n \frac{(d-1) !}{2}\right)^{1 /(d-1)} \log \gamma\right)=-\frac{1}{2} \log \gamma .
\end{gathered}
$$

Proof. We have

$$
h_{d}(k)=\frac{2}{(d-1) !}\left(k+\frac{d-1}{2}\right) \prod_{l=1}^{d-2}(k+l),
$$

where the product is interpreted to be equal to 1 if the upper range is strictly less than 1. By Proposition 3.3, we have

$$
\frac{\log \left|\log \gamma^{-1}\right|+\log \left(k+\frac{d}{2}\right)}{\log h_{d}(k)} \leq \frac{\log \left|\log s_{n}(J)\right|}{\log n} \leq \frac{\log \left|\log \gamma^{-1}\right|+\log \left(k+\frac{d}{2}\right)}{\log h_{d}(k-1)}
$$

Using (11), we obtain

$$
\lim _{k \rightarrow \infty} \frac{\log \left(k+\frac{d}{2}\right)}{\log h_{d}(k)}=\lim _{k \rightarrow \infty} \frac{\log \left(k+\frac{d}{2}\right)}{\log h_{d}(k-1)}=\frac{1}{d-1} .
$$

Combining (12) and (13), the assertion (8) follows.

Similarly, we have

$$
\frac{\left(k+\frac{d}{2}\right)\left|\log \gamma^{-1}\right|}{h_{d}(k)^{1 /(d-1)}} \leq \frac{\left|\log s_{n}(J)\right|}{n^{1 /(d-1)}} \leq \frac{\left(k+\frac{d}{2}\right)\left|\log \gamma^{-1}\right|}{h_{d}(k-1)^{1 /(d-1)}} .
$$

Since

$$
\lim _{k \rightarrow \infty} \frac{\left(k+\frac{d}{2}\right)}{h_{d}(k)^{1 /(d-1)}}=\lim _{k \rightarrow \infty} \frac{\left(k+\frac{d}{2}\right)}{h_{d}(k-1)^{1 /(d-1)}}=\left(\frac{(d-1) !}{2}\right)^{1 /(d-1)},
$$

equation (9) follows. 
It remains to establish (10). By Lemma 3.4, we have

$$
\begin{aligned}
\log s_{n}(J)+\left(n \frac{(d-1) !}{2}\right)^{1 /(d-1)} \log \gamma & =\left(\left(n \frac{(d-1) !}{2}\right)^{1 /(d-1)}-\left(k+\frac{d}{2}\right)\right) \log \gamma \\
& \leq\left(\left(h_{d}(k) \frac{(d-1) !}{2}\right)^{1 /(d-1)}-\left(k+\frac{d}{2}\right)\right) \log \gamma \\
& \leq\left(\frac{1}{d-1}\left(\sum_{l=1}^{d-2} l+\frac{d-1}{2}\right)-\frac{d}{2}\right) \log \gamma \\
& =-\frac{1}{2} \log \gamma .
\end{aligned}
$$

This proves

$$
\sup _{n \in \mathbb{N}}\left(\log s_{n}(J)+\left(n \frac{(d-1) !}{2}\right)^{1 /(d-1)} \log \gamma\right) \leq-\frac{1}{2} \log \gamma .
$$

To obtain equality we consider $s_{h_{d}(k)}(J)$ and again apply Lemma (3.4).

Finally, note that (10) is simply a restatement of (6) and (7).

\section{Singular values of arbitrary CANONICAL IDENTIFICATIONS}

We shall now show how to extend Proposition 3.5 to identifications of harmonic Bergman spaces on general open sets in $\mathbb{R}^{d}$. The main tool is the following construction.

Lemma 4.1. Let $U, V, W \subset \mathbb{R}^{d}$ be open with $U \subset V \subset W$. Then the operator

$$
T_{U}: h^{2}(V) \rightarrow h^{2}(W)
$$

defined by

$$
\left(T_{U} f, g\right)_{h^{2}(W)}=\int_{U} f(x) \overline{g(x)} d x
$$

is bounded with $\left\|T_{U}\right\| \leq 1$.

Proof. Indeed

$$
\left|\int_{U} f(x) \overline{g(x)} d x\right|^{2} \leq\left(\int_{U}|f|^{2}\right)\left(\int_{U}|g|^{2}\right) \leq\|f\|_{h^{2}(V)}^{2}\|g\|_{h^{2}(W)}^{2}
$$

implies that $T_{U}$ is well-defined and continuous with norm at most 1.

Definition 4.2. Let $\left\{\Omega_{n}\right\}_{1 \leq n \leq N}$ be a finite collection of open subsets of $\mathbb{R}^{d}$. A collection $\left\{\widetilde{\Omega}_{n}\right\}_{1 \leq n \leq N}$ of mutually disjoint open sets, with $\widetilde{\Omega}_{n} \subset \Omega_{n}$ for each $n$, is called a disjointification of $\left\{\Omega_{n}\right\}_{1 \leq n \leq N}$ if the symmetric difference $\left(\cup_{n=1}^{N} \Omega_{n}\right) \triangle\left(\cup_{n=1}^{N} \widetilde{\Omega}_{n}\right)$ is a Lebesgue null set.

We note that if a collection $\left\{\Omega_{n}\right\}_{1 \leq n \leq N}$ has the property that the boundary of each $\Omega_{n}$ is a Lebesgue null set, then a disjointification exists and can, for example, be obtained by setting 


$$
\widetilde{\Omega}_{1}=\Omega_{1}, \quad \widetilde{\Omega}_{n}=\operatorname{int}\left(\Omega_{n} \backslash\left(\bigcup_{i=1}^{n-1} \Omega_{i}\right)\right) \quad \text { for } \quad 2 \leq n \leq N .
$$

The usefulness of the operator $T_{U}$ is due to the following result.

Proposition 4.3. Let $\Omega \subset \mathbb{R}^{d}$ be open. Given open subsets $\Omega_{1}, \ldots, \Omega_{N}$ of $\Omega$, let

$$
J_{n}: h^{2}(\Omega) \rightarrow h^{2}\left(\Omega_{n}\right)
$$

be the canonical identification. If $\left\{\widetilde{\Omega}_{n}\right\}_{1 \leq n \leq N}$ is a disjointification of $\left\{\Omega_{n}\right\}_{1 \leq n \leq N}$, then the canonical identification

$$
J: h^{2}(\Omega) \rightarrow h^{2}\left(\bigcup_{n=1}^{N} \Omega_{n}\right)
$$

can be written as

$$
J=\sum_{n=1}^{N} T_{\widetilde{\Omega}_{n}} J_{n}
$$

where

$$
T_{\widetilde{\Omega}_{n}}: h^{2}\left(\Omega_{n}\right) \rightarrow h^{2}\left(\bigcup_{n=1}^{N} \Omega_{n}\right)
$$

is the operator defined in Lemma 4.1.

Proof. Let $f \in h^{2}(\Omega)$ and $g \in h^{2}\left(\bigcup_{n=1}^{N} \Omega_{n}\right)$. Then

$$
\begin{aligned}
\left(\sum_{n=1}^{N} T_{\widetilde{\Omega}_{n}} J_{n} f, g\right)_{h^{2}\left(\cup_{n} \Omega_{n}\right)} & =\sum_{n=1}^{N} \int_{\widetilde{\Omega}_{n}} f(x) \overline{g(x)} d x \\
& =\int_{\bigcup_{n} \Omega_{n}} f(x) \overline{g(x)} d x \\
& =(J f, g)_{h^{2}\left(\cup_{n} \Omega_{n}\right)}
\end{aligned}
$$

and the assertion follows.

Before proving the main result of this section we require some more terminology.

Definition 4.4. Let $\Omega_{1}, \Omega_{2} \subset \mathbb{R}^{d}$ be open with $\Omega_{2} \subset \Omega_{1}$. Let $N \in \mathbb{N}$. A finite collection $B_{1}, \ldots, B_{N}$ of balls is called a relative cover of the pair $\left(\Omega_{1}, \Omega_{2}\right)$ if the following two conditions hold:

(a) $\Omega_{2} \subset \bigcup_{n=1}^{N} B_{n}$;

(b) for each $1 \leq n \leq N$, there exists $\gamma_{n}>1$ such that $\bigcup_{n=1}^{N} B_{n}\left(\gamma_{n}\right) \subset \Omega_{1}$.

We call $N$ the size and $\left(\gamma_{1}, \ldots, \gamma_{N}\right)$ a scaling of the relative cover.

Given a relative cover $B_{1}, \ldots, B_{N}$ of $\left(\Omega_{1}, \Omega_{2}\right)$ with scaling $\left(\gamma_{1}, \ldots, \gamma_{N}\right)$, the vector

$$
\Gamma=\left(\log \gamma_{1}, \ldots, \log \gamma_{N}\right) \in \mathbb{R}_{+}^{N}
$$

is called the efficiency of the relative cover. We define

$$
\|\Gamma\|=\min _{1 \leq j \leq N}\left|\log \gamma_{j}\right|
$$


and for $k \in \mathbb{N}$,

$$
\|\Gamma\|_{k}=\left(\sum_{j=1}^{N}\left|\log \gamma_{j}\right|^{-k}\right)^{-1 / k}
$$

We note that, since $\Omega_{2}$ is relatively compact in $\Omega_{1}$, there always exists a relative cover for $\left(\Omega_{1}, \Omega_{2}\right)$.

We are now able to prove the main result of this section.

Theorem 4.5. Let $d \geq 2$ and let $\Omega_{1}, \Omega_{2} \subset \mathbb{R}^{d}$ be open with $\Omega_{2} \subset \Omega_{1}$. Suppose that $\left\{B_{n}\right\}_{1 \leq n \leq N}$ is a relative cover of $\left(\Omega_{1}, \Omega_{2}\right)$ of size $N$ with efficiency $\Gamma$. Then the canonical identification

$$
J: h^{2}\left(\Omega_{1}\right) \rightarrow h^{2}\left(\Omega_{2}\right)
$$

satisfies

$$
J \in E(c, 1 /(d-1)), \text { where } c=\left(\frac{(d-1) !}{2}\right)^{1 /(d-1)}\|\Gamma\|_{(d-1)},
$$

and

$$
|J|_{c, 1 /(d-1)} \leq N \exp (-\|\Gamma\| / 2) .
$$

Proof. Suppose that $\Gamma=\left(\log \gamma_{1}, \ldots, \log \gamma_{N}\right)$, where $\left(\gamma_{1}, \ldots, \gamma_{N}\right)$ is a scaling of $\left\{B_{n}\right\}_{1 \leq n \leq N}$. Let $\left\{\widetilde{\Omega}_{n}\right\}_{1 \leq n \leq N}$ be a disjointification of $\left\{B_{n}\right\}_{1 \leq n \leq N}$, and let

$$
T_{\widetilde{\Omega}_{n}}: h^{2}\left(B_{n}\right) \rightarrow h^{2}\left(\bigcup_{n=1}^{N} B_{n}\right) \quad(1 \leq n \leq N)
$$

denote the operator defined in Lemma 4.1. Consider the following canonical identifications:

$$
\begin{gathered}
\widetilde{J}_{n}: h^{2}\left(\Omega_{1}\right) \rightarrow h^{2}\left(B_{n}\left(\gamma_{n}\right)\right) \quad(1 \leq n \leq N), \\
J_{n}: h^{2}\left(B_{n}\left(\gamma_{n}\right)\right) \hookrightarrow h^{2}\left(B_{n}\right) \quad(1 \leq n \leq N), \\
\widetilde{J}: h^{2}\left(\bigcup_{n=1}^{N} B_{n}\right) \rightarrow h^{2}\left(\Omega_{2}\right) .
\end{gathered}
$$

By Proposition 4.3 we have

$$
J=\sum_{n=1}^{N} \widetilde{J} T_{\widetilde{\Omega}_{n}} J_{n} \widetilde{J}_{n}
$$

Since $\|\widetilde{J}\| \leq 1$ and $\left\|\widetilde{J}_{n}\right\| \leq 1$, while $\left\|T_{\widetilde{\Omega}_{n}}\right\| \leq 1$ by Lemma 4.1, we conclude, by Propositions 2.2 and 3.5 , that for $1 \leq n \leq N$,

$$
\widetilde{J} T_{\widetilde{\Omega}_{n}} J_{n} \widetilde{J}_{n} \in E\left(c_{n}, 1 /(d-1)\right), \quad \text { with } c_{n}=\left(\frac{(d-1) !}{2}\right)^{1 /(d-1)} \log \gamma_{n}
$$

and

$$
\left|\widetilde{J} T_{\widetilde{\Omega}_{n}} J_{n} \widetilde{J}_{n}\right|_{c_{n}, 1 /(d-1)} \leq \exp \left(-(1 / 2) \log \gamma_{n}\right) .
$$

The assertion now follows from Proposition 2.2. 


\section{BOUNDED OPERATORS ON SPACES OF HARMONIC FUNCTIONS}

We are now able to prove the main result which gives explicit upper bounds for the eigenvalues of bounded operators on the space $h(\Omega)$ of harmonic functions on an open set $\Omega \subset \mathbb{R}^{d}$. In order to specify a topology on $h(\Omega)$ we define, for each $\Omega^{\prime} \subset \mathbb{R}^{d}$ with $\Omega^{\prime} \subset \Omega$, the following seminorm on $h(\Omega)$

$$
p_{\Omega^{\prime}}(f):=\sqrt{\int_{\Omega^{\prime}}|f(x)|^{2} d x} .
$$

If $\left\{\Omega_{n}\right\}_{n \in \mathbb{N}}$ is a collection of open subsets of $\mathbb{R}^{d}$ such that

(i) $\Omega_{n} \subset \Omega_{n+1}$ for every $n \in \mathbb{N}$,

(ii) $\bigcup_{n \in \mathbb{N}} \Omega_{n}=\Omega$,

then $\left\{p_{\Omega_{n}}\right\}$ forms a directed system of seminorms which turns $h(\Omega)$ into a Fréchet space, whose topology is equivalent to the topology of uniform convergence on compact subsets of $\Omega$. Moreover, since each canonical identification $h^{2}\left(\Omega_{n+1}\right) \rightarrow$ $h^{2}\left(\Omega_{n}\right)$ is nuclear by Theorem 4.5, the space $h(\Omega)$ is nuclear.

A study of other kinds of harmonic function spaces can be found in [6].

Recall that a subset $S$ of a topological vector space $E$ is bounded if for each neighbourhood $U$ of 0 , we have $S \subset \alpha U$ for some $\alpha>0$. A linear operator $L: E \rightarrow E$ is bounded if it takes a neighbourhood of zero into a bounded set. In order to formulate the main result, we require the following definition.

Definition 5.1. Let $\Omega, \Omega^{\prime} \subset \mathbb{R}^{d}$ be open with $\Omega^{\prime} \subset \Omega$. A linear operator $L$ : $h(\Omega) \rightarrow h(\Omega)$ is called $\Omega^{\prime}$-bounded if for every $\Omega^{\prime \prime} \subset \Omega$ there is a positive constant $k$ such that

$$
p_{\Omega^{\prime \prime}}(L f) \leq k p_{\Omega^{\prime}}(f) \quad \text { for every } f \in h(\Omega) .
$$

Clearly, a linear operator $L: h(\Omega) \rightarrow h(\Omega)$ is bounded if and only if it is $\Omega^{\prime}$-bounded for some $\Omega^{\prime} \subset \Omega$.

We shall now discuss some natural examples of bounded operators on $h(\Omega)$ for plane domains $\Omega$. We identify the complex plane $\mathbb{C}$ with $\mathbb{R}^{2}$. By a conformal map on $\Omega$ we mean a holomorphic map $\varphi: \Omega \rightarrow \mathbb{C}$ such that the derivative $\varphi^{\prime}$ has no zero, in which case, the differential

$$
d \varphi(z): \mathbb{R}^{2} \rightarrow \mathbb{R}^{2}
$$

at each $z \in \Omega$ is a linear isomorphism since $\operatorname{det} d \varphi(z)=\left|\varphi^{\prime}(z)\right|^{2} \neq 0$. Hence $\varphi$ is a local diffeomorphism on $\Omega$. Let $\Pi=\left\{z \in \mathbb{R}^{2}: \Im z>0\right\}$ be the upper half-plane in $\mathbb{R}^{2}$. We can define a conformal mapping from $\Pi$ onto a proper region $\Omega_{2} \subset \Pi$. For instance, the conformal map

$$
\psi: z \in U \mapsto\left(\frac{1+z}{1-z}\right)^{2} \in \Pi
$$

sends the semicircular $\operatorname{disc} U \subset \Pi$, centred at 0 with radius 1 , onto $\Pi$. Note that $\psi$ is one-to-one on $\mathrm{U}$. Hence the conformal map $\varphi=\psi^{-1}+2 i$ sends $\Pi$ onto the translation $\Omega_{2}=U+2 i$ of $U$ with $\Omega_{2} \subset \Pi$. Another example is the SchwarzChristoffel transformation

$$
\varphi(z)=\int_{0}^{z} \frac{d t}{\sqrt{\left(1-t^{2}\right)\left(1-t^{2} / 4\right)}} \quad(z \in \Omega),
$$


which maps $\Pi$ conformally onto a rectangle in $\Pi$ (cf. [1, p. 231]).

Example 5.2. Let $\Omega \subset \mathbb{R}^{2}$ be a domain and let $\varphi: \Omega \rightarrow \Omega^{\prime}$ be a conformal bijection whose image $\Omega^{\prime}$ satisfies $\Omega^{\prime} \subset \Omega$. Since harmonic functions on plane domains are real parts of holomorphic functions, one can define a composition operator $L_{\varphi}: h(\Omega) \rightarrow h(\Omega)$ by

$$
L_{\varphi}(f)=f \circ \varphi \quad(f \in h(\Omega)) .
$$

Then $L_{\varphi}$ is $\Omega^{\prime}$-bounded. To see this, let $\Omega^{\prime \prime}$ be open with $\Omega^{\prime \prime} \subset \Omega$. Then we have, via a change of variable,

$$
\begin{aligned}
p_{\Omega^{\prime \prime}}\left(L_{\varphi} f\right)^{2} & =\int_{\Omega^{\prime \prime}}|f \circ \varphi(x)|^{2} d x=\int_{\varphi\left(\Omega^{\prime \prime}\right)}|f(x)|^{2}\left|\operatorname{det} d \varphi^{-1}(x)\right| d x \\
& \leq k \int_{\varphi\left(\Omega^{\prime \prime}\right)}|f(x)|^{2} d x \\
& \leq k p_{\Omega^{\prime}}(f)^{2},
\end{aligned}
$$

where $k=\sup _{x \in \varphi\left(\Omega^{\prime \prime}\right)}\left|\operatorname{det} d \varphi^{-1}(x)\right|<\infty$. Thus $L_{\varphi}$ is $\Omega^{\prime}$-bounded.

More generally, if $\varphi: \Omega \rightarrow \Omega^{\prime}$ is any conformal map between plane domains with $\Omega^{\prime} \Subset \Omega$, then a local change of variables together with a compactness argument shows that $L_{\varphi}$ is bounded in this case as well.

For open sets $\Omega$ in Euclidean space $\mathbb{R}^{d}$ of dimension greater than 2, one can construct bounded composition operators $L_{\varphi}$ on $h(\Omega)$ analogous to the above example, but the choice of $\varphi: \Omega \rightarrow \Omega$ is more delicate. A smooth map $\varphi: \Omega \rightarrow \Omega$ for which the composition operator $L_{\varphi}: h(\Omega) \rightarrow h(\Omega)$ is well-defined is called a harmonic morphism. Harmonic morphisms between Riemannian manifolds have been characterized and widely studied. We refer to 3] for details and examples.

We are now ready to prove the main result.

Theorem 5.3. Let $\Omega$ be open in $\mathbb{R}^{d}(d>1)$ and let $L: h(\Omega) \rightarrow h(\Omega)$ be $\Omega^{\prime}$-bounded for some $\Omega^{\prime} \subset \Omega$. If $\Omega^{\prime \prime}$ is open with

$$
\Omega^{\prime} \lessdot \Omega^{\prime \prime} \lessdot \Omega
$$

and such that $\left(\Omega^{\prime \prime}, \Omega^{\prime}\right)$ has a relative cover of size $N$ and efficiency $\Gamma$, then

$$
\lambda(L) \in \mathcal{E}(c, 1 /(d-1)) \text { with }|\lambda(L)|_{c, 1 /(d-1)} \leq K N \exp (-\|\Gamma\| / 2),
$$

where

$$
c=\frac{d-1}{d}\left(\frac{(d-1) !}{2}\right)^{1 /(d-1)}\|\Gamma\|_{(d-1)}
$$

and

$$
K=\sup \left\{p_{\Omega^{\prime \prime}}(L f) \mid f \in h(\Omega), p_{\Omega^{\prime}}(f) \leq 1\right\} .
$$

Proof. Define the following canonical identifications

$$
\begin{gathered}
J_{1}: h(\Omega) \rightarrow h^{2}\left(\Omega^{\prime \prime}\right), \\
J_{2}: h^{2}\left(\Omega^{\prime \prime}\right) \rightarrow h^{2}\left(\Omega^{\prime}\right) .
\end{gathered}
$$


Clearly, $J_{1}$ and $J_{2}$ are continuous. Let $\overline{J_{2} J_{1} h(\Omega)}$ be the closure of $J_{2} J_{1} h(\Omega)$ in the Hilbert space $h^{2}\left(\Omega^{\prime}\right)$ and let $P: h^{2}\left(\Omega^{\prime}\right) \rightarrow \overline{J_{2} J_{1} h(\Omega)}$ be the natural projection. Since $L$ is $\Omega^{\prime}$-bounded, the linear map

$$
\left.f\right|_{\Omega^{\prime}} \in J_{2} J_{1} h(\Omega) \mapsto L f \in h(\Omega) \quad(f \in h(\Omega))
$$

is well defined and bounded, and therefore extends to a bounded linear map

$$
\widetilde{L}: \overline{J_{2} J_{1} h(\Omega)} \rightarrow h(\Omega) \text {. }
$$

We now observe that $L$ admits the following factorisation

$$
L=\widetilde{L} P J_{2} J_{1} .
$$

By Pietsch's principle of related operators (see [9, Satz 1 and Satz 2]) it follows that

$$
\lambda(L)=\lambda\left(\widetilde{L} P J_{2} J_{1}\right)=\lambda\left(J_{1} \widetilde{L} P J_{2}\right) .
$$

But since $J_{1} \widetilde{L} P: h^{2}\left(\Omega^{\prime}\right) \rightarrow h^{2}\left(\Omega^{\prime \prime}\right)$ is bounded with norm $K$ and since, by Theorem 4.5, we have $J_{2} \in E\left(c^{\prime}, 1 /(d-1)\right)$ with $\left|J_{2}\right|_{c^{\prime}, 1 /(d-1)} \leq N \exp (-\|\Gamma\| / 2)$, where

$$
c^{\prime}=\left(\frac{(d-1) !}{2}\right)^{1 /(d-1)}\|\Gamma\|_{(d-1)}
$$

it follows by Proposition 2.2 that $\lambda\left(J_{1} \widetilde{L} P J_{2}\right) \in \mathcal{E}\left((d-1) c^{\prime} / d, 1 /(d-1)\right)$ with $\left|J_{1} \widetilde{L} P J_{2}\right|_{(d-1) c^{\prime} / d, 1 /(d-1)} \leq K N \exp (-\|\Gamma\| / 2)$. Thus $\lambda(L)$ has the desired properties.

An immediate consequence of the previous theorem is the following analogue of Grothendieck's Remarque 9 mentioned in the introduction.

Corollary 5.4. Let $\Omega$ be open in $\mathbb{R}^{d}(d>1)$ and let $L: h(\Omega) \rightarrow h(\Omega)$ be a bounded linear operator. Then $\lambda(L) \in \mathcal{E}(1 /(d-1))$.

Example 5.5. As in the discussion before Example 5.2, let $\varphi: \Pi \rightarrow \Omega_{2}$ be the one-to-one conformal map from the upper half-plane $\Pi$ onto the semicircular disc $\Omega_{2}$, centred at the point $2 i$ with radius 1 . Consider the composition operator $L_{\varphi}: h(\Pi) \rightarrow h(\Pi)$ defined in Example 5.2. Let $\Omega_{1}$ be the open disc $B_{\gamma, 2 i}$ centred at the point $2 i$ with radius $1<\gamma<2$. Then we have $\Omega_{2} \subset \Omega_{1} \lessdot \Pi$ while $B_{2,2 i} \not \subset \Pi$. By Definition 4.4, the singleton $\left\{B_{1,2 i}\right\}$ is a relative cover of $\left(\Omega_{1}, \Omega_{2}\right)$ with optimal scaling $\gamma$, and efficiency $\Gamma=\log \gamma$. We have $d-1=1$ for $\Pi \subset \mathbb{R}^{2}$. Hence $\|\Gamma\|=\log \gamma=\|\Gamma\|_{1}$ and $c=\frac{1}{4} \log \gamma$, as in Theorem 5.3 which gives the following eigenvalue asymptotics

$$
\left|\lambda_{n}\left(L_{\varphi}\right)\right| \leq K \exp (-(\log \gamma) / 2) \exp (-(\log \gamma) n / 4)
$$

where

$$
K=\sup _{z \in \varphi\left(B_{\gamma, 2 i}\right)} \sqrt{\left|\operatorname{det} d \varphi^{-1}(z)\right|}=\sup _{z \in \varphi\left(B_{\gamma, 2 i}\right)}\left|\frac{4-8 i+z}{(1+2 i-z)^{3}}\right| .
$$

In particular, we see that

$$
\lambda_{n}\left(L_{\varphi}\right)=O\left(\gamma^{-n / 4}\right)
$$

for every $\gamma<2$. 


\section{ACKnowledgement}

We would like to thank an anonymous referee for useful comments and suggestions that helped to improve the presentation of this article.

\section{REFERENCES}

[1] L.V. Ahlfors, Complex Analysis, McGraw-Hill, New York, 1966.

[2] S. Axler, P. Bourdon, and W. Ramey, Harmonic Function Theory, Springer-Verlag, NewYork, 2001.

[3] P. Baird and J.C. Wood, Harmonic morphisms between Riemannian manifolds, LMS Monograph 29, Oxford Univ. Press, Oxford, 2003.

[4] O.F. Bandtlow, Resolvent estimates for operators belonging to exponential classes, Integr. Equ. Oper. Theory 61 (2008) 21-43.

[5] O.F. Bandtlow and O. Jenkinson, Explicit eigenvalue estimates for transfer operators acting on spaces of holomorphic functions, Adv. Math. 218 (2008) 902-925.

[6] C-H. Chu, Harmonic function spaces on groups, J. London Math. Soc. 70 (2004) 182-198.

[7] A. Grothendieck, Produits tensoriels topologiques et espaces nucléaires, Mem. Amer. Math. Soc. 16, 1955.

[8] C. Müller, Spherical harmonics, Lecture Notes in Mathematics 17, Springer-Verlag, Berlin, 1966.

[9] A. Pietsch, Zur Fredholmschen Theorie in lokalkonvexen Räumen, Studia Math. 22 (1963) 161-179.

[10] W. Rudin, Real and complex analysis, McGraw-Hill, New York, 1966.

Oscar F. Bandtlow, School of Mathematical Sciences, Queen Mary, UniverSITY OF LONDON, LONDON E1 4NS, UK

Cho-Ho Chu, School of Mathematical Sciences, Queen Mary, University of LONDON, LONDON E1 4NS, UK 